\title{
SIMPLE FACE THERMAL FEATURES FOR GENDER DISCRIMINATION
}

\author{
Georgia Koukiou and Vassilis Anastassopoulos \\ Electronics Laboratory Physics Department, University of Patras, Greece
}

\begin{abstract}
A very simple approach is proposed for gender discrimination using thermal infrared images of the persons' face. The selected features are actually based on the mean value of the pixels of specific locations on the face. It is proved that the discrimination is feasible either by simple visualization in the feature space or by using a relatively simple neural network for this purpose. All cases of persons from the used database, males and females, are correctly distinguished based on the mean value of the employed locations of the face. Classification results are verified using two conventional approaches, namely: a. the simplest possible neural network so that generalization is achieved along with successful discrimination between all persons and $b$. the leave-one-out approach to demonstrate the classification performance on unknown persons using the simplest classifiers possible.
\end{abstract}

\section{KEYWORDS}

Thermal Infrared, Face Recognition, Gender Discrimination, Neural Networks.

\section{INTRODUCTION}

The approach used in this correspondence for feature extraction was based on a thorough comparison between the available images in the thermal infrared region. The thermal infrared region of the electromagnetic spectrum which lies between 7 and 13 microns, is the region in which radiate all bodies with typical temperature that of the environment. Infrared signatures of the face describe face temperature distribution which depends on physiological and psychological condition of the person.

Infrared images acquired from the face of males and females differ significantly in specific locations. Accordingly, the used features should incorporate this type of information. Thus, the mean value of the pixels in four different locations of the face is used to form the feature vector. This feature vector is fed into Neural Network structures to perform gender identification. In this work, we seek for the simplest neural structure to perform successful gender identification and simultaneously we examine the suitability of the derived features to support correct identification even in the case of unknown persons present. For this purpose the leave-one-out method is employed. The classification performance of the neural structures is very efficient giving very good gender separability in the feature space, which is proved by the distinct locations of the clusters of males and females in the feature space.

The work is organized as follows. Work related to the presented method is extensively exposed in section 2. In section 3 the infrared data employed for gender identification are described. In the 
next section the feature extraction approach is outlined and the feature space is demonstrated. Gender identification performance is carried out in section 5, while the conclusions are drawn in section 6 .

\section{RELATED WORK}

Various techniques have been presented in the past regarding gender discrimination. In [1] features are combined using fusion techniques from visible and infrared images for gender recognition. The presented technique is very complicated and the features used from specific locations on the face in case of infrared images are not conceptual prominent, while a similar approach for feature extraction has been proposed in the past [2] for drunk person discrimination. Furthermore, thermal features using eye signatures were used with successful results in intoxication identification [3]. Gender recognition using body-based images from visible and thermal cameras was carried out in [4]. The approach is basically employing movement detection and classification algorithms. The possibility of deducing gender from face images obtained in the near-infrared (NIR) and thermal (THM) spectra is elaborated in [5] by means of local binary pattern histogram (LBPH) features. In [6-7] methods for gender and ethnicity identification are described with small emphasis on thermal signatures. In [8] a combination of visible and infrared cameras is employed based on body images and neural networks for gender identification.

Other approaches have also been developed for gender discrimination based on handwriting, voice, walking movements and fingerprints. Specifically, in [9] a system is presented to predict gender of individuals from offline handwriting samples. The technique relies on extracting a set of textural features from handwriting samples of male and female writers and training multiple classifiers to learn to discriminate between the two gender classes. The features include local binary patterns, histogram of oriented gradients, statistics computed from gray-level cooccurrence matrices and features extracted through segmentation-based fractal texture analysis. The work in [10] presents a study to predict gender of individuals from scanned images of their handwritings. The proposed methodology is based on extracting a set of features from writing samples of male and female writers and training classifiers to learn to discriminate between the two. Writing attributes like slant, curvature, texture and legibility are estimated by computing local and global features. The study in [11] investigates the relative importance of temporal and spectral cues in voice gender discrimination and vowel recognition by normal-hearing subjects listening to an acoustic simulation of cochlear implant speech processing and by cochlear implant users. The results suggest that both spectral and temporal cues contribute to voice gender discrimination and that temporal cues are especially important for cochlear implant users to identify the voice gender when there is reduced spectral resolution.

An adaptive three-mode PCA framework was presented in [12] for recognizing gender from walking movements. Prototype female and male walkers are initially decomposed into a subspace of their three-mode components (posture, time, gender). Then is assigned an importance weight to each motion trajectory in the sub-space and have the model automatically learn the weight values (key features) from labeled training data. In [13] it is revealed that an informationrich latent fingerprint has not been used to its full potential. The content present in the sweat left behind - namely the amino acids - can be used to determine physical such as gender of the originator. As a result, it is possible to focus on the biochemical content in the fingerprint using a biocatalytic assay, coupled with a specially designed extraction protocol, for determining gender rather than focusing solely on the physical image. 


\section{INFRARED DATABASE USED}

The thermal infrared images required to assess the proposed gender identification method have been obtained by our research team during a systematic and well organized experimental procedure. Researchers from our university were asked to participate in the experiment. The Thermal Vision Micron/A10 infrared camera was employed for image acquisition. The resolution of the camera is 160x128 pixels and is sensitive in the thermal infrared region of the electromagnetic spectrum i.e. from 7.5 to $13.0 \mu \mathrm{m}$. At the middle of this region of wavelengths i.e. at $10 \mu \mathrm{m}$, a perfect black body with mean temperature around 300 degrees Kelvin, radiates its maximum according to the Wien Law [14]. The human body as being in the same temperature with the environment i.e. 300 degrees Kelvin, radiates in this exact region of wavelengths.

The participants in the experiment were healthy, calm and in normal physical and psychological condition. No illness or fever, no psychological stress, other pathological reason or any kind of body exercises were recorded for any one of the participants. Thus, the thermal images of the face were suitable for comparisons between males and females without having been affected by other factors.

The thermal infrared images were recorded in a well organized database. The database contains information regarding the age, the weight and the sex of the participant. In each acquisition, a sequence of 50 frames was acquired from each person with a sampling period of $100 \mathrm{msec}$ between the frames.

In order to secure smooth experimental procedure that would lead to reliable data acquisition the participants were asked to be present in the room of the experiment half an hour earlier and to keep calm during the experimental procedure. Simultaneously, the temperature of the room where measurements took place was kept between 23 and 25 degrees Celsius (almost 300 Kelvin). No physical light was used in the room of the experiment. The distance of each face from the camera was kept almost $30 \mathrm{~cm}$ for all persons so that the acquired images could be compared. The images of eight females and 8 males from the database were employed in this present gender discrimination procedure. In Figure 1 are shown a thermal image from the face of a male (left) along with one from a female (right).
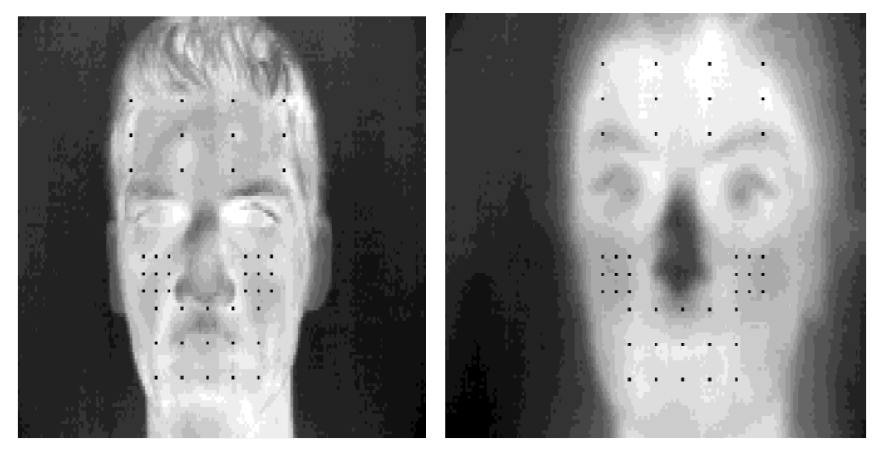

Figure 1: Infrared images of the face of a man (left) and a woman (right). According to the gray level of the regions depicted with the black dots the two genders differentiate significantly in their thermal representation. 


\section{Feature EXTRACTION AND GENDER DisCRIMINATION}

In the thermal infrared region of the electromagnetic spectrum (7-13 microns) the face transmits electromagnetic radiation as an almost perfect black body and the amount of transmitted energy depends on the physiological condition of the person and is irrelevant of the external artificial lighting. The features to be selected for gender discrimination have to describe the temperature distribution on the face of the persons and obviously this temperature distribution map has to be different between males and females. In order to check this temperature distribution on the face of the persons the values of the pixels in four specific regions of the face are recorded.

In Figure 1 are shown the faces of a man and a woman obtained from our database. The four inspected regions on each face are: the forehead, the left cheek, the right cheek and the region of the mouth. By simple observation it is obvious that the region of the forehead has different brightness for the females compared to the corresponding of the males. The same happens for the region of mouth as well. Consequently, each person's face is represented in a 4-dimentional space by means of these four values. In practice, if in each face the intensity (temperature) of the forehead is considered as the value of reference for the other locations, then the feature space is of 3 dimensions and the persons can be represented in this space with three values each. This fact is graphically depicted in Figure 2 for eight men (cross) and eight women (diamond) of our database. In this representation can be easily realized that the two populations form distinct clusters in the feature space that can be separated by a relatively simple decision boundary (discriminant function) not necessarily linear.

The data available for the eight males and eight females are given in Tables 1 and 2. For each person a four-point column is provided. The data are given in order the reader to be able to test and verify their capability in gender classification.

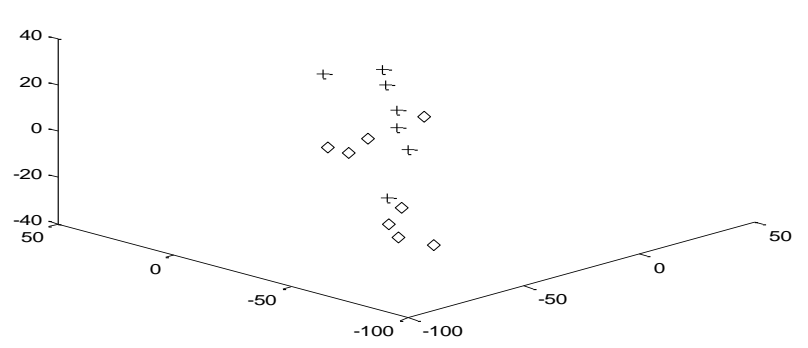

Figure 2. The 3-D feature space. Eight men (crosses) and eight women (diamonds) from our database are depicted. Males and females can be easily separated except the female (diamond) on the upper right.

Table 1. In each column are given the values for each male in the feature space.

\begin{tabular}{|c|c|c|c|c|c|c|c|}
\hline $\mathbf{1}$ & $\mathbf{2}$ & $\mathbf{3}$ & $\mathbf{4}$ & $\mathbf{5}$ & $\mathbf{6}$ & $\mathbf{7}$ & $\mathbf{8}$ \\
\hline 208 & 227 & 195 & 222 & 204 & 198 & 171 & 221 \\
\hline 168 & 197 & 196 & 205 & 204 & 173 & 200 & 224 \\
\hline 168 & 205 & 206 & 208 & 212 & 208 & 193 & 227 \\
\hline 208 & 198 & 205 & 217 & 207 & 212 & 208 & 214 \\
\hline
\end{tabular}


Table 2. In each column are given the values for each female in the feature space.

\begin{tabular}{|c|c|c|c|c|c|c|c|}
\hline $\mathbf{1}$ & $\mathbf{2}$ & $\mathbf{3}$ & $\mathbf{4}$ & $\mathbf{5}$ & $\mathbf{6}$ & $\mathbf{7}$ & $\mathbf{8}$ \\
\hline 234 & 236 & 227 & 230 & 225 & 225 & 227 & 215 \\
\hline 168 & 170 & 183 & 154 & 208 & 202 & 182 & 191 \\
\hline 176 & 174 & 185 & 142 & 232 & 218 & 216 & 183 \\
\hline 214 & 211 & 203 & 212 & 205 & 216 & 221 & 223 \\
\hline
\end{tabular}

\section{Gender Classification Results}

Gender classification results were carried out using two different conventional classification approaches. Firstly, a neural network is employed in order to examine the separability of the feature space into two subspaces corresponding to the two gender classes and simultaneously to study the degree of difficulty in drawing a discriminating hyper plane among the two classes. After that the leave-one-out approach is used to demonstrate the classification performance of the employed features on unknown persons.

\subsection{Classification using neural networks}

The classification attempt started by testing feed forward neural networks having a significant number of neurons in their 3 layers. The training algorithm was the conventional back propagation procedure. All MATLAB available routines were used.

Two different goals we tried to achieve during training. The first one was to select a neural structure capable to converge fast to a minimum giving the best gender classification result. For this purpose we started with a network having a relatively large number of perceptrons i.e. 21 neurons in the input layer, 11 neurons in the hidden layer and 1 neuron in the output layer (binary output - male or female). This network was found very effective in classifying the 16 persons.

The second goal was the selected neural structures to meet the property of generalization. This means to be capable after training to correctly classify the new incoming vectors representing persons of unknown gender. For this purpose a low number of epochs was selected in the training procedure.

Lowering gradually the number of neurons in the input and hidden layers we found that even a very simple neural structure of 3-2-1 can satisfactorily classify all the 16 samples in the 4-D feature space. Even if this simple structure was converging to a minimum after several attempts, it was preferred compared to larger ones since it was very effective in classifying unknown samples according to the leave-one-out approach given in the next subsection. For the reader to be able to test our results, the weights and the biases of this simple structure are given in Tables 3 to 5. In Table 3 is given the information regarding the 3 neurons of the input layer. In Table 4 the relative information for the hidden layer is given, while in Table 5 the weights and the bias for the output neuron are provided. 
Table 3. Weights and biases values for the 3 neurons of the input layer

\begin{tabular}{|c|c|c|c|}
\hline & Neuron 1 & Neuron 2 & Neuron 3 \\
\hline W1 & -0.3419 & -0.2219 & 0.0195 \\
\hline W2 & 2.8386 & -0.2261 & 0.0045 \\
\hline W3 & -1.3043 & 0.0305 & 0.0221 \\
\hline W4 & -1.0688 & 0.3757 & 0.0101 \\
\hline bias & 0.3403 & 1.1603 & 0.6821 \\
\hline
\end{tabular}

Table 4. Weights and biases values for the 2 neurons of the hidden layer

\begin{tabular}{|c|c|c|}
\hline & Neuron 1 & Neuron 2 \\
\hline W1 & -1.3745 & -1.7958 \\
\hline W2 & -0.4105 & -1.5866 \\
\hline W3 & 1.0303 & 0.0669 \\
\hline bias & 1.7587 & -1.5691 \\
\hline
\end{tabular}

Table 5. Weights and bias values for the output neuron

\begin{tabular}{|c|c|c|c|}
\hline & W1 & W2 & bias \\
\hline Output Neuron & 0.3320 & -2.9791 & 0.9287 \\
\hline
\end{tabular}

\subsection{Leave-one-out approach}

The Leave-one-out method is employed to verify that the used neural structure behave appropriately with the available data in the classification procedure. i.e. the classifier is built using in the training phase all the data except one, and is expected in the test phase to be able to classify correctly the excluded information. This is actually the generalization property which requires the classifier not to be over trained on the available data, so that be able to classify correctly the new incoming vectors representing persons of unknown gender.

For this purpose a neural structure of size 3-2-1 is trained using 15 of the persons and then is tested whether the excluded person is correctly classified. This procedure was repeated 16 times, each time excluding one of the persons. In all cases, the devised neural structures classified with success the remaining person. The classification goal (classification error) achieved during the training procedure by each of the 16 neural structures was very small which reveals that this small neural network adapts fast to the needs of the problem. In Table 6 the classification goals for all 16 structures are given. 
Table 6. Classification goals for all 16 structures devised for the leave-one-out method

\begin{tabular}{|c|c|c|}
\hline $\begin{array}{c}\text { Neural } \\
\text { structure }\end{array}$ & $\begin{array}{c}\text { Person } \\
\text { excluded }\end{array}$ & $\begin{array}{c}\text { Classification } \\
\text { goal }\end{array}$ \\
\hline 1 & 1st male & 0.001 \\
\hline 2 & 2nd male & 0.009 \\
\hline 3 & 3rd male & 0.002 \\
\hline 4 & 4th male & 0.038 \\
\hline 5 & 5th male & 0.009 \\
\hline 6 & 6th male & 0.003 \\
\hline 7 & 7th male & 0.001 \\
\hline 8 & 8th male & 0.001 \\
\hline 9 & 1st female & 0.001 \\
\hline 10 & 2nd female & 0.008 \\
\hline 11 & 3rd female & 0.030 \\
\hline 12 & 4th female & 0.003 \\
\hline 13 & 5th female & 0.090 \\
\hline 14 & 6th female & 0.130 \\
\hline 15 & 7th female & 0.009 \\
\hline 16 & 8th female & 0.004 \\
\hline
\end{tabular}

We have to mention at this point that only in the 16 th case in Table 6 , the neural structure of size 3-2-1 neurons achieved after many attempts a convergence so that it was able to correctly classify the excluded 16th person or otherwise the 8th female. However, this behaviour was expected since according to the feature space depicted in Figure 2, this person was eventually deviating from the distribution of the cluster corresponding to females.

\section{Conclusions}

Gender discrimination is feasible using thermal images from the face of a person. This is possible since the thermal map of the face differs from males to females. This fact is evident if the temperature of the forehead is compared to that of other locations on the face. In this work sixteen persons were employed to carry out the experimental procedure. The feature selected was very simple since it consists of the mean value of the pixel in specific locations of the face.

Examination of the feature space, as it was depicted in Figure 2, shows that there is a significant clustering tendency of the two gender populations. Consequently, the used features are potentially good candidates for gender classification.

In the experimental procedure it is proved that the clusters corresponding to the male and female subjects can be easily separated using a simple neural structure. Experimentally, various neural structures were tested starting from complex ones and concluding to the simplest with 3-2-1 neurons in the corresponding layers. This leads to the conclusion that the classification problem is easily manageable.

Furthermore, it was shown using the leave-one-out method that the classification can be successful for data for which the classifier has not been trained with. In all 16 cases the person excluded each time from the training procedure was correctly recognized by the corresponding neural structure. The data provided throughout the paper can help the reader to verify the results and compare with his approaches. The proposed gender classification procedure can be combined with other features derived from the persons' face, such as geometric and relative distance features in order to further improve gender classification procedures. 


\section{REFERENCES}

[1] Shangfei Wang, Zhen Gao, Shan He, Menghua He and Qiang Ji, (2016), "Gender recognition from visible and thermal infrared facial images”, Multimedia Tools and Applications, Volume 75, Issue 14, pp. 8419-8442.

[2] Georgia Koukiou and Vassilis Anastassopoulos, (2012), "Drunk person identification using thermal infrared images", Int. J. Electronic Security and Digital Forensics, Vol. 4, No. 4, pp. 229-243..

[3] Georgia Koukiou, and Vassilis Anastassopoulos, (2016), "Drunk Person Screening using Eye Thermal Signatures", J. Forensic Sci, Vol. 61, No. 1, pp. 259-264.

[4] Dat Tien Nguyen and Kang Ryoung Park, (2016), "Body-Based Gender Recognition Using Images from Visible and Thermal Cameras", Sensors (Basel), 16(2), p.156.

[5] Cunjian Chen and Arun RossLane, (2011), "Evaluation of Gender Classification Methods on Thermal and Near-infrared Face Images", Proc. of International Joint Conference on Biometrics (IJCB), Washington DC, USA.

[6] Xiaoguang Lu, Hong Chen and Anil K. Jain, (2006), "Multimodal Facial Gender and Ethnicity Identification", International Conference on Biometrics, ICB 2006: Advances in Biometrics pp. 554561, Hong Kong, China.

[7] Mohamed, Abouelenien., Verónica, Pérez-Rosas., Rada, Mihalcea., and Mihai, Burzo., (2017), "Multimodal gender detection", 19th International Conference on Multimodal Interaction, CMI'17, November 13-17, Glasgow, UK.

[8] Dat Tien. Nguyen,, Ki Wan, Kim,. Hyung, Gil Hong., Ja Hyung, Koo., Min Cheol, Kim. and Kang Ryoung, Park., (2017), "Gender Recognition from Human-Body Images Using Visible-Light and Thermal Camera Videos Based on a Convolutional Neural Network for Image Feature Extraction”, Sensors, 17(3), 637; https://doi.org/10.3390/s17030637

[9] Mahreen Ahmed, Asma Ghulam Rasool, Hammad Afzal, Imran Siddiqi, (2017), "Improving handwriting based gender classification using ensemble classifiers", Expert Systems with Applications, Volume 85, Pages 158-168.

[10] Imran Siddiqi, Chawki Djeddi, Ahsen Raza, Labiba Souici-meslati, "Automatic analysis of handwriting for gender classification”, Pattern Analysis and Applications, volume 18, pages 887-899 (2015).

[11] Qian-Jie Fu, Sherol Chinchilla, John J. Galvin, (2004), “The Role of Spectral and Temporal Cues in Voice Gender Discrimination by Normal-Hearing Listeners and Cochlear Implant Users", Journal of the Association for Research in Otolaryngology, volume 5, pages 253-260.

[12] J.W. Davis, Hui Gao, (2004), "Gender Recognition from Walking Movements using Adaptive ThreeMode PCA”, : 2004 IEEE Conference on Computer Vision and Pattern Recognition Workshop, 27 June-2 July 2004, Washington, DC, USA, USA, DOI: 10.1109/CVPR.2004.354.

[13] Crystal Huynh, Erica Brunelle, Lenka Halámková, Juliana Agudelo, Jan Halámek, (2015), “Forensic Identification of Gender from Fingerprints", Anal. Chem., 87, 22, 11531-11536.

[14] N.A. Diakides, J.D. Bronzino, (2008), Medical Infrared Imaging, Chapter 6: Physiology of Thermal Signals CRC Press, Taylor \& Francis Group, New York. 


\section{AUTHORS}

Georgia Koukiou (B.Sc., M.Sc., Ph.D) was born in Patras. She received the B.Sc. degree in Physics in 2004, the M.Sc. in Electronics and Computers in 2006 and her Ph.D. in Digital Image and Signal Processing in 2014 all from University of Patras. She is a member of the research team of Digital Image and Signal Processing of Electronics Laboratory, Physics Department in the University of Patras. She is currently working in Face Identification using Thermal Infrared, Biomedical imaging, Remote Sensing, SAR imaging, Ground Penetrating Radar methods and Information Fusion specializing in Decision Fusion methods. She has 20 publications in International Journals and Conferences.

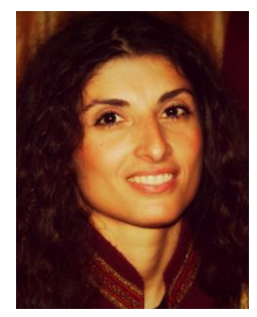

Dr. Koukiou is a PostDoc. Scholar of IKY. She is currently giving lectures on Electronics, Programming, Signal and Image Processing, Pattern Recognition and Remote Sensing at University of Patras and University of Thessaly.

Prof. Vassilis Anastassopoulos was born in Patras, Greece, in 1958. He received the B.Sc. degree in Physics in 1980 and his Ph.D. in Electronics in 1986, both from the University of Patras, Greece. He worked for two years in Canadian Scientific Institutions. He is an academic staff member in Physics Department, University of Patras, Greece from 1987. He is a Professor from 2005. He was the Head of Physics Department, University of Patras, for the biennium 2001-2003 and 2005-2007, and the Vice Rector for Strategic Research Planning and Development in the University of Patras from 2006 to 2010.

His publication record contains over 100 journal and conference papers with over than 1500 citations. His research interests are within the scope of Digital Signal Processing, Image Processing, Radar Signal Processing and Remote Sensing with emphasis in Multi-spectral, SAR and Infrared Imagery, Signal Detection, Pattern Recognition with emphasis in Handwritten Analysis, and Information Fusion including Image Fusion, Decision Fusion and Sensor Fusion Architectures. Lately, he was involved in image processing techniques for Astro-particle Physics.

(C) 2020 By AIRCC Publishing Corporation. This article is published under the Creative Commons Attribution (CC BY) license. 